\title{
NOTAS
}

\section{Los Fueros de Aragón. Una versión romance de mediados del siglo XIII}

\author{
The Fueros of Aragon. A Romance version \\ from the Mid-13 ${ }^{\text {th }}$ Century
}

\author{
Miguel Carabias Orgaz \\ IES El Greco (Toledo)
}

\begin{abstract}
RESUMEN: Presentamos una versión hasta ahora inédita de los Fueros de Aragón, aprobados en las Cortes de Huesca de 1247, que nos ha llegado en un documento de mediados del siglo XIII. Se trata, por tanto, del testimonio más antiguo conocido de la llamada Compilatio Minor. El manuscrito, conservado fragmentariamente, contiene diez fueros pertenecientes al segundo libro, están escritos en romance aragonés y coinciden casi literalmente con la versión latina del texto foral.
\end{abstract}

Palabras clave: Fueros de Aragón, Compilación de Huesca, Compilatio Minor, derecho medieval, romance aragonés.

ABSTRACT: We present a hitherto unknown version of the Fueros of Aragon, approved by the Cortes of Huesca in 1247 , that came to us in the form of a mid- $13^{\text {th }}$ Century document. This is, therefore, the oldest known testimony of the so-called Compilatio Minor. The fragmentarily-kept manuscript comprises ten fueros belonging to the second book which are written in Aragonese Romance language and are almost identical to the Latin version of the jurisdictional text.

Keywords: Fueros of Aragon, Huesca Compilation, Compilatio Minor, medieval law, Aragonese dialect.

\section{LA COMPILACIÓN DE Huesca DE 1247 y LOS Fueros dE ARAGÓN}

Hacia el mes de enero de 1247 Jaime I de Aragón reunía las Cortes en su ciudad de Huesca con la intención de fijar —así nos lo explica el prólogo Cum 
de foris - un texto legal que remediara la falta de documentos forales fiables. Durante aquellas Cortes se procedió a la lectura, corrección y expurgo de muy diversos materiales ya existentes, sobre todo los contenidos en el llamado Fuero de Jaca, y luego se añadieron otros nuevos. Una vez aprobada la que conocemos como Compilación de Huesca, se encomendó al obispo Vidal de Canellas que recogiera aquellos fueros en un libro. El resultado, sin embargo, quedó plasmado en dos obras diferentes: una extensa recopilación de fueros, glosados y ampliados por Vidal de acuerdo con el Derecho romano, la cual sería conocida como Compilatio Maior ${ }^{1}$ y rechazada como texto legal; y por otro lado la llamada Compilatio Minor, con similar ordenación que la Maior pero mucho más reducida y ajustada al derecho tradicional aragonés, la cual tuvo vigencia legal hasta el siglo XVIII y es el texto que hoy conocemos como el de los Fueros de Aragón².

De la Compilatio Minor tenemos hoy una versión latina recogida en doce códices manuscritos, todos ellos de entre los siglos XIV y XV. Éstos contienen una redacción de los Fueros muy similar, presentando escasas variantes y coincidiendo en lo esencial con las ediciones impresas que aparecieron a partir de $1476^{3}$.

No sucede lo mismo, sin embargo, con las escasas fuentes en lengua romance hasta ahora conocidas. Todas ellas presentan notables diferencias entre sí y también con respecto a la redacción latina:

— El códice 458 de la Biblioteca Nacional de Madrid. Es un manuscrito de comienzos del siglo XIV editado y estudiado por Tilander (1937), quien ya observó que el texto recogido en él se aparta con frecuencia de la versión latina, coincidiendo en estos casos con el Vidal Mayor.

— El códice 7 (olim 207) de la Biblioteca Universitaria de Zaragoza, de comienzos del XV. Lo editaron Lacruz Berdejo (1945) y Bergua (1950). Fue considerada por Lalinde (1976: 67) como la versión más veraz de las que conocía, por ser más fiel que las otras al texto latino, pese a que reconoció en ella bastantes errores de copia.

- La versión de los Fueros conservada en el Archivo Municipal de Miravete de la Sierra, un códice de finales del siglo XIII o comienzos del XIV. Fue editada por Antonio Gargallo (1992), y presenta notables va-

\footnotetext{
${ }^{1}$ No se ha conservado el texto latino original. Únicamente contamos con una versión romance conservada en copia del siglo XIV. Es el llamado Vidal Mayor, cuya edición llevó a cabo Gunnar Tilander (1956).

${ }^{2}$ La autoría de la Compilatio Minor ha sido muy discutida. Si para algunos autores, como Gonzalo Martínez Díez (1980), cabe dudar de que Vidal de Canellas participase en su redacción, otros autores como Antonio Pérez Martín (1985) o Jesús Delgado Echeverría $(1989,1997)$ defienden que a él se debe la ordenación de dicho texto.

${ }^{3}$ Una edición crítica de las diversas fuentes conservadas del texto latino ha sido publicada por Antonio Pérez Martín (2010).
} 
riantes con respecto a la versión latina. Incluye diversos fueros que no se hallan en ésta, y omite otros tantos.

- El códice J.J.N.N. de los Archivos Nacionales de París. Es un documento del siglo XIV que fue ya descrito por Molho (1964: XXII-XXIII) y publicado años después por Pérez Martín (1999). Presenta importantes coincidencias con respecto al códice 458 de Madrid, aunque no mantiene la división en libros y contiene frecuentes errores.

- Cabría añadir, por último, la versión fragmentaria contenida en un documento del Archivo Notarial de Tarazona, editada por Edo Quintana (1962), copia del siglo XV que contiene únicamente el prólogo Nos Iacobus y parte de los ocho primeros fueros. Coincide, a grandes rasgos, con el texto del manuscrito de la Biblioteca Nacional de Madrid.

Como puede verse, son importantes las divergencias entre los testimonios romances señalados, lo cual ha venido dificultando la tarea de fijar el texto. Además, todos estos testimonios han llegado a nosotros a través de manuscritos de los siglos XIV y XV, es decir, muy posteriores a la probable fecha de redacción (1247).

En las últimas décadas se ha venido planteando la necesidad de determinar cuál de las versiones conservadas cabe ser considerada como la más próxima al texto original, aunque desafortunadamente hasta ahora no se ha podido llegar a una respuesta convincente. Tal como ya señalasen hace años Feenstra (1961) y Lalinde (1976), aún carecemos de una edición del texto que nos permita un análisis preciso de la Compilación de Huesca.

Lalinde supuso que la versión latina era la más cercana a la redacción de 1247, y por ese motivo dio prioridad al códice zaragozano entre los textos romances (Lalinde, 1976: 67). Enseguida veremos que tal vez no estuviera desencaminado. Por su parte, Pérez Marín (1999: 13-15) y algún otro autor han considerado más fiel la versión contenida en el códice de Miravete, que suponen anterior a la redacción latina conservada ${ }^{4}$.

El texto que presento a continuación, una versión romance de los Fueros totalmente desconocida hasta ahora, tal vez arrojará algo de luz en el complejo entramado textual de esta obra. Aunque se trata de un testimonio breve y fragmentario — contiene apenas diez fueros pertenecientes al segundo libro-, ofrece un doble atractivo: en primer lugar, el haber llegado a nosotros en un documento datable hacia 1250 , es decir, muy próximo a la fecha de redacción de los Fueros; por otro lado, que en cuanto a su contenido y estructura coincide casi exactamente con la versión latina.

\footnotetext{
${ }^{4}$ Entre los otros autores que han defendido recientemente la prioridad del códice de Miravete cabe mencionar a Jesús Morales Arrizabalaga (2007: 32-48).
} 


\section{FIJACIÓN Y TRANSMISIÓN DEL TEXTO}

El texto que aprobaron las Cortes, la Compilatio Minor, seguramente fue redactado y corregido en lengua romance para que pudieran comprenderlo todos aquéllos que debían dar su aprobación. Así parece desprenderse del siguiente pasaje del Vidal Mayor (Tilender, 1956: 522):

La antiguidat es diuulgada por iuditio de fuero por unas palauras apropriadas [...] tanto son las palauras estranias del latín o encara tantas son estranias del lengoage de Aragón, que non pueden ser espuestas aqueillas palauras de rafez en latín si non por palabras que son ditas acerqua d'aqueillas, nin los qui fablan en su romantz pueden entender aillí, si non fueren mayestrados por sotil enseynnança $[\ldots]$

Es un indicio de que los Fueros se vertieron del romance al latín, la lengua "oficial" que no podía ser comprendida por quienes sólo fablan en su romantz y no eran mayestrados por sotil enseynnança. Al habla vulgar debían de pertenecer esas palauras apropriadas de la antiguidat que precisaban de alguien que las "declarase"; es decir, que existía una terminología jurídica vinculada a conceptos nacidos en romance que no podían ser traducidos al latín si no era por medio de glosas y explicaciones (Frago: 1989, 106).

Una vez aprobados los fueros, el rey los haría leer públicamente y luego encargaría a un jurista que añadiera los títulos y tradujera el texto al latín, que era la lengua de la cancillería. Así explicó ya en su momento Gunnar Tilander (1937: XXVIII-XXIX) el proceso de redacción del texto foral de 1247, tomando como referencia lo sucedido años después con respecto a los fueros introducidos por Jaime II, que encomendó esta tarea a Jimeno Pérez de Salanova, justicia de Aragón: De romancio in latinum translatis, et sub debitis titulis collocatis.

Por ese motivo, es bastante significativo que el texto que aquí presento no contenga ninguna clase de títulos o rúbricas, a diferencia del pasaje latino correspondiente. Si a ello añadimos el hecho de que ha llegado a nosotros en un manuscrito de mediados del siglo XIII, y que además coincide casi puntualmente con la versión latina considerada "oficial", no sería aventurado pensar que nos encontramos ante un testimonio de la redacción primigenia, o que al menos está muy cerca de ella.

Sobre esta primera versión romance, poco después, se elaboraría el texto latino que conocemos, llevando a cabo una traducción casi literal y añadiendo las rúbricas pertinentes. El carácter "oficial” de la versión latina explicaría, en parte, la homogeneidad de los manuscritos que de la misma se han conservado.

Con posterioridad, años o décadas más tarde, debido seguramente a necesidades de la práctica jurídica, irían surgiendo diversas traducciones romances 
destinadas a facilitar la comprensión de la versión latina "oficial". El texto latino sería romanceado, por tanto, aunque no con demasiada fidelidad.

Así pues, el proceso de transmisión del texto partió de una primitiva redacción en lengua vulgar, que se tradujo al latín, y del texto latino surgieron con posterioridad varias y muy diversas versiones romances.

Naturalmente, hoy resulta difícil reconocer huellas de la traducción o las traducciones a que fue sometido el texto durante aquellos años. Entre otras cosas, porque las fronteras linguiísticas en el ámbito románico del siglo XIII, especialmente en la lengua escrita, eran aún muy difusas. Por su parte, el latín hacía tiempo que se empleaba tan solo en determinados ámbitos, como el jurídico, y no era precisamente un latín depurado ni demasiado correcto, sino que mezclaba fórmulas jurídicas latinas y términos propios de la lengua vernácula, los cuales no siempre tenían un equivalente culto. A todo ello hay que añadir el hecho de que, en la elaboración del texto de la Compilación de Huesca, se utilizaron muy diversos materiales, los cuales probablemente fueron redactados en diversas lenguas y terminaron dejando su impronta lingüística en muchos pasajes.

Pese a todo, podemos reconocer algunos rasgos lingüísticos que hacen pensar en la versión latina de los Fueros como una traducción. Se advierte cierta tendencia al literalismo de tipo estructural, pues hallamos estructuras calcadas que reproducen uno a uno los elementos de la oración traducida, incluso rompiendo con los mecanismos sintácticos propios del latín. También hay un literalismo de tipo semántico, pues algunos términos adquieren en el texto un valor que no es el propio de la lengua latina, y hay además un buen número de vocablos tomados de la lengua romance que aparecen allí latinizados. Estos dos fenómenos quedan sobradamente plasmados en el texto: guerrae, medianeto, spondaleriis, cabeçaleriis, pignale, forum, torna, infantio, batalla, etc.

Por otro lado, es probable que, a consecuencia de las disposiciones introducidas por Jaime II en las Cortes de Zaragoza del año 1300, el texto foral experimentara algunos cambios a comienzos del siglo XIV. Éstos consistieron principalmente en una reordenación de los materiales ya existentes, que quedaron distribuidos en ocho libros, y a los que se añadió uno nuevo que sería el noveno. Sin embargo, estoy convencido de que los cambios no afectaron al contenido ni a la estructura interna de los libros. Tal como ha señalado Delgado Echeverría (1997: 48), simplemente se llevó a cabo la unión del segundo y el tercer libro, dejando intacto todo lo demás. Es decir, que el texto aprobado en Huesca en 1247 permaneció prácticamente inalterado.

Ahora bien, ¿cómo se explica que las versiones romances posteriores presenten tal cantidad de variantes y se aparten de manera considerable del texto latino?... Tal vez se deba, en primer lugar, a que éstas no recogían una versión "oficial" de los Fueros, algo que, tras su aprobación, correspondía exclusivamente al texto latino. Es decir, que se trataba de copias romanceadas destinadas a aclarar o glosar 
la versión en latín. Algo similar a lo que pudo llegar a ocurrir con la Compilatio Maior, y concretamente con su versión romance, el Vidal Mayor. Incluso se ha venido considerando que alguno de estos testimonios, como el códice de la Biblioteca Nacional, representa una "versión intermedia" entre la Compilatio Minor y la Maior (Delgado, 1989: 80) o un "resumen de Vidal Mayor" (Pérez, 1979: 8). Debemos recordar que en el periodo medieval la traducción no se ajustaba en absoluto a la noción actual de fidelidad. La frontera entre las palabras del autor y las del traductor era con frecuencia difusa y, por tanto, no había una distinción clara entre el texto original y su traducción. En ese sentido cabe interpretar el siguiente párrafo del Vidal Mayor (Tilander, 1956: 521-522):

[...] en el declaramiento de las quoales palauras conuiene que aqueill qui las declara que se departa de la propriedat d'aqueillas o usar de ordenamiento de palauras que es rudo del todo et indigesta, ço es non clara, empero quar millor cosa es saquar la uerdat por palauras rudas que aillenar la uerdat del sono de las palauras et del seso que deue auer, andando en uano por flores rethoricas, es assaber por palabras afeitadas. Mas aproueitable cosa es entender a las palauras de propriedat que son a pro que desuiar de la uirtud de la dreiturera sentencia $[\ldots]$

Se proponía, como vemos, que el jurista buscase la manera más adecuada de dar a entender las palabras del fuero, lo cual había de hacerse por medio de comentarios, glosas y una traducción libre del texto.

Además, debemos tener presente que los testimonios romances de que estamos hablando han llegado a nosotros a través de copias al menos cincuenta años posteriores al texto que se aprobó en Huesca, y que por tanto se vieron sometidos a un proceso de transmisión salpicado de errores.

No sucede lo mismo con la versión de los Fueros que aquí presento. En este sentido, podríamos mencionar como ejemplo una cláusula que se halla en el fuero número 7 de nuestro manuscrito. En él, al hablar de los testigos y del modo en que deben utilizarse, encontramos la siguiente afirmación:

[...] si son de otro regno, puede se aiudar en medianedo.

Con ese mismo sentido se redacta el texto latino:

[...] si sunt alterius regni, non potest se iuvare de eis, nisi in medianeto.

Sin embargo, el códice de la Biblioteca Nacional, que se redacta a la vista del texto latino, altera completamente su sentido:

[...] si son de otro regno, non se puede aiudar dellos en medianedo.

Todas estas variantes afectarán, no solo a la redacción del texto, sino también a la ordenación de los fueros. Como podrá verse en la tabla de concordan- 
cias que incluyo a continuación, nuestro manuscrito, que llamaré $C A$, coincide exactamente con el texto latino de la Compilación de Huesca $(\mathrm{CH})$, al igual que el códice zaragozano $(Z)$, que presenta solo variantes en su redacción. En cambio, las versiones de Miravete $(M)$ y de la Biblioteca Nacional $(F A)$ se van apartando hacia ordenaciones intermedias entre la Compilatio Minor y el Vidal Mayor $(V)$.

\begin{tabular}{|c|c|c|c|c|c|}
\hline $\mathrm{CA}$ & $\mathrm{CH}$ & $\mathrm{Z}$ & $\mathrm{M}$ & $\mathrm{FA}$ & $\mathrm{V}$ \\
\hline 1 & 2.10 .6 & 103 & 131 & 106 & 3.25 \\
\hline 2 & 2.10 .7 & 104 & 132 & 107 & 3.26 \\
\hline 3 & 2.11 .1 & 105 & 143 & 108 & 3.35 \\
\hline 4 & 2.11 .2 & 106 & 133 & 112 & 3.27 \\
\hline 5 & 2.11 .3 & 107 & 134 & 109 & 3.30 \\
\hline 6 & 2.11 .4 & 108 & 135 & 111 & 3.29 \\
\hline 7 & 2.11 .5 & 109 & 137 & 113 & 3.31 \\
\hline 8 & 2.11 .6 & 110 & 138 & 114 & - \\
\hline 9 & 2.11 .7 & 111 & 139 & 116 & 3.32 \\
\hline 10 & 2.11 .8 & 112 & 140 & 117 & 3.33 \\
\hline
\end{tabular}

\section{DESCRIPCIÓN DEL MANUSCRITO}

Se trata de un folio manuscrito sobre vitela, algo deteriorado, que hallé formando parte de la encuadernación que protegía un legajo con documentación notarial, de mediados del siglo XVI, relativa a la villa de Cantavieja (Teruel). El documento ha quedado depositado en la Biblioteca Nacional de España. Presenta unas dimensiones de $150 \times 210 \mathrm{~mm}$, y la caja de escritura es de $115 \times 176 \mathrm{~mm}$. El texto está copiado a línea tirada y presenta veintiséis renglones tanto en el recto como en el vuelto. El contenido aparece distribuido en diez párrafos, uno de ellos incompleto, y no presenta rúbricas ni títulos. Los párrafos, salvo el que está incompleto, aparecen encabezados por iniciales rojas y azules. Una posterior corrección del texto suprimió y añadió algunas palabras. Los añadidos aparecen interlineados, mediante reclamo, y se han hecho con tinta de color más claro. También se observa que algunos pasajes fueron borrados o raspados, escribiéndose encima con la misma tinta de color claro que la de los añadidos. Es muy escasa la puntuación, reducida exclusivamente al uso del punto (.) y del guión inclinado (/) para indicar la separación de palabras al final del renglón.

La letra del documento es gótica textual. Siguiendo la opinión del profesor José Manuel Ruiz Asencio, a quien manifiesto desde estas líneas mi más since- 
ra gratitud, podemos detectar algunos rasgos que nos permiten datar el manuscrito: el predominio del ángulo y el contraste de gruesos y finos indican que se trata de letra gótica, pero con rasgos de la primera etapa, pues alternan la $d$ minúscula y la $d$ uncial, tanto en posición inicial como en el interior de palabra; no siempre se hace unión de curvas contrapuestas y se utiliza la $s$ alta al final de palabra. Todo ello sitúa el documento hacia mediados del siglo XIII y, por tanto, en fechas muy cercanas a la de redacción de los Fueros de Aragón, esto es, 1247.

En lo que se refiere a la lengua empleada en el texto, podemos decir que, aunque presenta características comunes a diversos romances peninsulares del siglo XIII, destacan algunos rasgos que cabe poner en relación con el primitivo romance aragonés: la conservación del grupo inicial latino KL- (clama), el posesivo de tercera persona lures, la conservación de -D- intervocálica (fidança), o formas como juge, parçoneros, etc.

\section{CONCLUSIONES}

Aunque nos encontramos ante un pasaje breve y fragmentario, el texto aquí presentado merece especial atención, en primer lugar, por tratarse del testimonio documental más antiguo hasta ahora conocido de los Fueros de Aragón. Tal y como se desprende de un primer análisis paleográfico, el manuscrito seguramente fue copiado hacia 1250 , poco después de que el texto foral se aprobara en las Cortes de Huesca.

En segundo lugar, es significativo que el texto de nuestro manuscrito coincida fielmente con la versión latina considerada "oficial", tanto en su contenido como en la ordenación de los fueros. Aunque a comienzos del siglo XIV se habían llevado a cabo algunas modificaciones en la Compilatio Minor, éstas apenas afectaron al contenido de los fueros ya existentes, como puede comprobarse al cotejar nuestro manuscrito, de mediados del siglo XIII, con los códices latinos de los siglos XIV y XV.

Es más que probable, por lo tanto, que nos hallemos ante el único testimonio conservado de la redacción primitiva en lengua romance, sobre la cual se llevó a cabo una traducción al latín que terminaría convirtiéndose en el texto "oficial" de los Fueros.

Posteriormente surgirían diversas versiones romances, pero éstas siguieron ya al texto latino. Serían traducciones más o menos libres, muy divergentes entre sí, pues no tuvieron un carácter "oficial", sino que estaban destinadas probablemente a aclarar y facilitar la interpretación de los fueros redactados en latín. 
A la vista de todo lo que acabo de mencionar, me atrevería a hablar de tres grupos o tradiciones en los testimonios conservados:

- La más antigua y más fiel al texto original, de la que son testimonio nuestro manuscrito $(C A)$ y la redacción latina de la Compilación de Huesca $(\mathrm{CH})$. El códice zaragozano $(\mathrm{Z})$, aunque copiado ya en el siglo $\mathrm{XV}$ y con abundantes errores, se aproxima al texto latino y puede ponerse en relación con esta tradición.

— El códice de Miravete $(M)$. Se aparta de manera significativa de la versión latina, aunque no tanto como el manuscrito 458 de la Biblioteca Nacional.

— La versión representada por el códice madrileño de la Biblioteca Nacional $(F A)$, que podríamos considerar una versión intermedia entre la Compilatio Minor y el Vidal Mayor (V). Dentro de este grupo cabría incluir el códice parisino J.J.N.N. y el fragmentario de Tarazona, que coinciden en gran medida con el texto del códice de Madrid.

\section{CRITERIOS DE EDICIÓN}

Transcribo el texto fielmente, aunque adaptándolo a la moderna puntuación y separando las palabras según criterios actuales. También el uso de las mayúsculas se ajusta a las normas ortográficas vigentes. No he tenido en cuenta la diferencia entre $s$ alta y $s$ de doble curva, aunque sí respeto los casos de elisión en el artículo, individualizándolo por medio de apóstrofo.

Las lagunas o carencias del texto debidas al deterioro en el documento son suplidas entre corchetes [ ]. El texto superfluo o tachado figura entre paréntesis ( ). Las palabras que he añadido para completar el sentido del texto aparecen entre paréntesis agudos $<>$. Las adiciones que hizo el corrector del manuscrito, tanto las interlineales como las que sustituyen al texto borrado, las transcribo entre líneas oblicuas convergentes $\backslash /$.

Las abreviaturas se han desarrollado en cursiva. El signo tironiano aparece transcrito como et. En cuanto a los numerales, mantengo la expresión numérica (I, II, etc.) cuando así aparece en el manuscrito.

Reservo las notas a pie de página para dar cuenta de aquellas particularidades que pueden contribuir a conocer mejor el documento y aclarar el sentido del texto. 


\section{TEXTO}

Fol. 1r

...[predi]cho porq[ue] non pudo passar agua o muert o enfermeda[t] et por $\mathrm{gu}[\mathrm{e}] \mathrm{ra}<\mathrm{a}$ la $>$ que fuer[e] $<\mathrm{o}>$ otro uerdadero o sufficient enbargamiento, non seya auido por [u]encido, mas seya dado a elli otro dia a recebir los testigos, et la tercera uocacion et el encendimiento del fuego no aya lugar.

\section{[2]}

Torna a batalla no a lugar entre cristiano et jodio et moro, mas qual se [defendie]re del otro (por propio) por propio sagrament en toda razon, enpero quiscuno jure por su ley. Enpero si el cristiano contra el jodio a carta sobre alguna cosa et lo quisiere prouar, por II testigos, et assaber cristiano et jodio son i menester. Et el jodio exament ${ }^{5}$ contra el cristiano, p[rueua c]on jodio et cristiano. Exament contra'l moro, en toda cosa prueua con cristiano et moro, et el contrario. El moro exa misma ment, prueua en toda cosa contra'l judio con moro et jodio.

\section{$[3]^{6}$}

$\mathrm{M}[\mathrm{ui}] \operatorname{tas}$ ueg[ada]s alguno de qual condicion que seya non quisiere fer testimonio a [ot]ro omne de qual condicion quiere que seya, el sennor o la cort de aquel lugar o [habit]are aquel testigo constrigan a el fer el testimonio.

\section{[4]}

De pla[zo] firmado lentre algunos que uengan/ delant el juge. Si el uno uinie[re] [d]elant el juge et no el otro, estando al dicho $<$ del $>$ juge ${ }^{7}$ sobre la pres[enta]cion o el desestamiento del uno de ellos, et el su dicho aya u[alor] de II testigos.

Si el senno[r] de alguna uilla a aprouar alguna cosa contra alguno, se[gun]t del fuero non puede nin deue en aquella uilla o es el sennor ldar a/ su[s b]asall[o]s ni otros de su signo o de su honor len testigos, mas/ puede dar infançones leales et hauientes ${ }^{8}$ sennorio. Et si non puede auer [tale]s quales ju[z]ga [d]el fuero, reciba del otro del qual se clama I jura.

\footnotetext{
${ }^{5}$ Una estructura adverbial semejante la encontramos en las llamadas redacción $C$ y redacción E publicadas por Molho (1964: 454 y 597): Et yssament proua lo judeu de tot fayt. Tal vez podría ponerse en relación con el antiguo romance occitano.

${ }^{6}$ La versión latina presenta la rúbrica De testibus cogendis.

${ }^{7}$ Aunque puede tratarse de un error de copia, la elisión de la preposición de en formas equivalentes al genitivo posesivo es frecuente en el habla de comarcas del Alto Aragón.

${ }^{8} \mathrm{Da}$ la impresión de que el copista añadió con posterioridad la $h$ inicial, cuyo valor sería meramente etimológico.
} 
Fol. 1v

De aquel que conpra9 ${ }^{9}$ o recibe en pennos alguna heredat; aquella mism[a] fidança et los testigos Isegunt del fuero/ deuen seer de aquella uilla o es la heredat, et deuen saber et ueder aquella misma hereda[t], que por cierto sepan de qual heredat son fidanças et testigos, que si assi feito non fuere, el conprador o el que el pennal recibe puede ${ }^{10}$ a ella de cierto perderla, qual la fidança et los testigos non saben ont son fidanças o testigos. Exament, qui conpra o en pennos recibe, si non recibe testigos et fidança del heredat puede a ella por [1']qual fallimiento perder, et si assi pudieren seer auidos los testigos o las fidanças del lugar o de la uilla o fuere la heredat, seya auida. O si auer no la pudieren, seya auida del regno, de qual que lugar, seyan buenos et leales o de cruz.

\section{[7]}

De espondaleros, cabeceleros o testigos; aquel que los faze o los estableçe, en qual que lugar los faze a ellos, i \se/ aura ajuld/ar de ellos ${ }^{11}$, encara si son de otro regno puede se aiudar ${ }^{12}$ en medianedo.

[8]

De aquestos cristianos que testimonian por jodios et por moros sobre qual quiere cosa, despues que aquel mismo cristiano jurare, despues por ninguna manera no lo podra el otro tornar a ninguna batalla.

\section{[9]}

De aquel que por mandamiento del judez aduze su[s t]estigos sobre alguna razon, et los testigos offrecen fidanças de [lu]res juras ad aquel \contral el qual quisieren testimoniar, ni esta por ellos qual cosa los testigos deuen fer. Si aquel contra al qual son aduchos non quiere recebir a ellos, demientre que fueren a la fidança abondantes, desent non son tenidos testimoniar ni dar fidanças de sus juras, qual el fuero cunplieron.

Como algun infançon o otro sobre alguna demanda al culpable alguno aduze testigos, Isegunt del fuero/ non puede dar testigos parçoneros en la misma demanda.

\footnotetext{
${ }^{9}$ Desarrollo la abreviatura con la grafía $n$ pues parece ser esta la forma utilizada habitualmente en el texto delante de consonante labial.

${ }^{10}$ Parece que el copista trató de modificar la última letra de esta palabra, de manera que podríamos leer pueda.

${ }^{11}$ Quien corrigió el texto enmienda esta cláusula utilizando la misma tinta de color claro que usa otras veces. Originalmente se leía: i aurá a jurar de ellos. En la corrección añadió la forma pronominal se y escribió una $d$ sobre la $r$ de jurar.

${ }^{12}$ Da la sensación de que el mismo copista ha alargado el rasgo de la $i$ para que sea ajudar.
} 
Fol. 1r

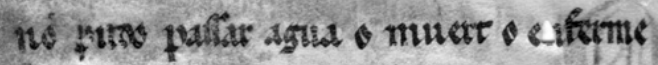

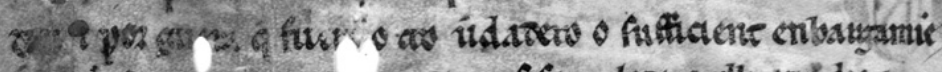

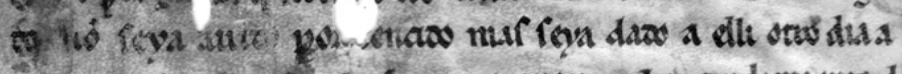

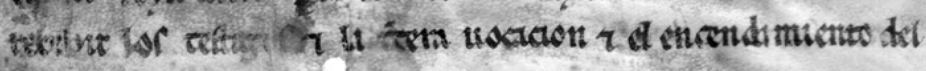
firge no $\operatorname{sgn}$ thosat?

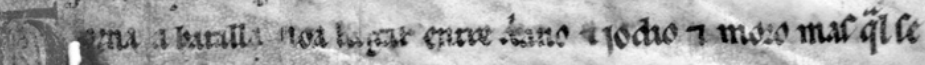

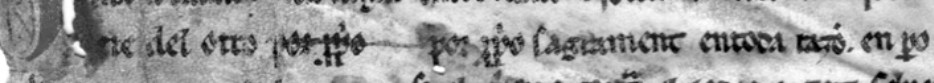

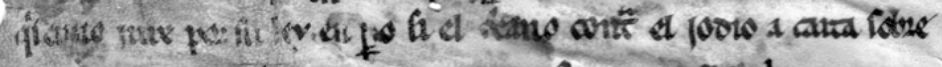

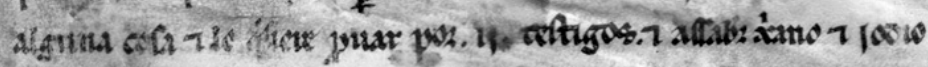

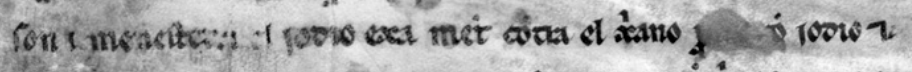

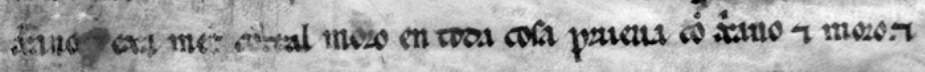

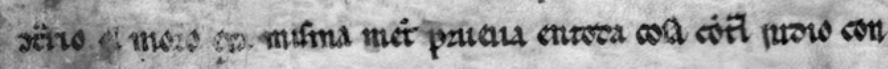

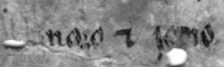

(f) पy

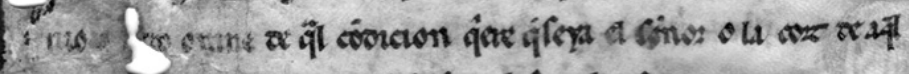

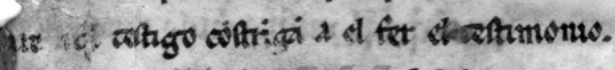

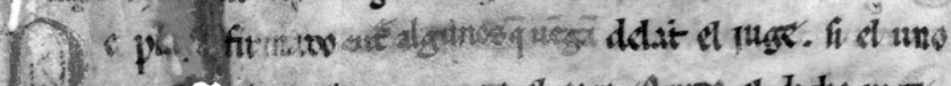

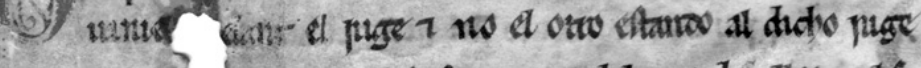
fols: ha ing

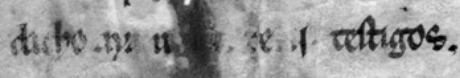

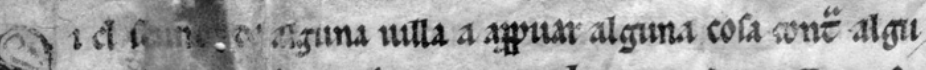

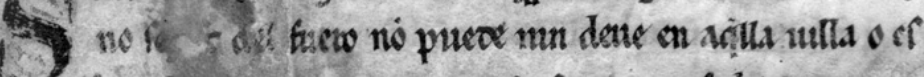

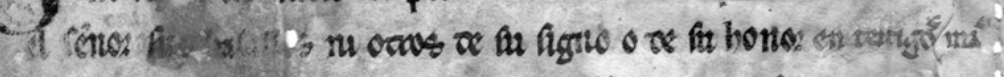

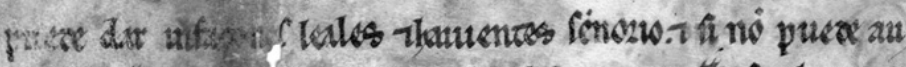

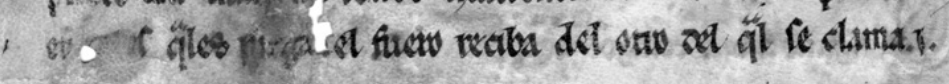
Iิ: 
Fol. 1v

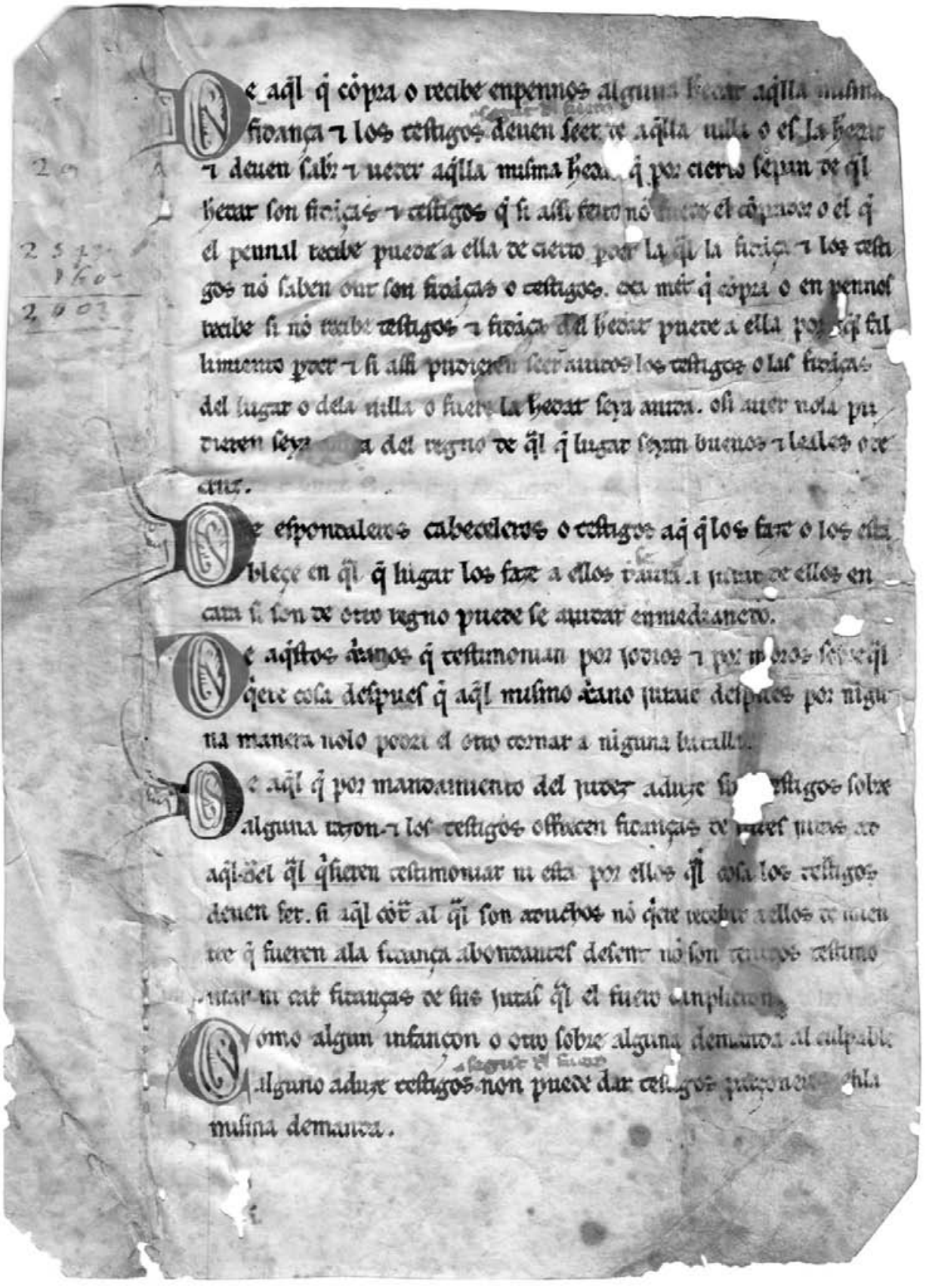




\section{BIBLIOGRAFÍA}

Bergua Camón, Jesús (1950): "Fueros de Aragón de 1265 a 1381. Versión romanceada contenida en el manuscrito 207 de la Biblioteca Universitaria de Zaragoza", Anuario de Derecho Aragonés, V, pp. 455-575.

Delgado Echeverría, Jesús (1989): "Vidal Mayor, un libro de fueros del siglo XIII", en Agustín Ubieto Arteta (coord.), Vidal Mayor, Huesca, Diputación Provincial, Instituto de Estudios Altoaragoneses, pp. 45-81.

Delgado Echeverría, Jesús (1997): Los Fueros de Aragón, Colección "Mariano de Pano y Ruata", Zaragoza, Caja de ahorros de la Inmaculada de Aragón.

Edo Quintana, Antonio (1962): "Un manuscrito incompleto de los Fueros de Aragón, en el protocolo notarial de Tarazona”, Anuario de Derecho Aragonés, XI, pp. 179-188.

Feenstra, Robert (1961): "Vidal Mayor. Traducción aragonesa de la obra In excelsis Dei Thesauris de Vidal de Canellas, editada por Gunnar Tilender", Zeitschrift der Savigny-Stiftung für Rechtsgeschichte, 78, pp. 343-352.

Frago Gracia, Juan A. (1989): "El marco filológico del Vidal Mayor”, en Agustín Ubieto Arteta (coord.), Vidal Mayor, Huesca, Diputación Provincial, Instituto de Estudios Altoaragoneses, pp. 83-112.

Gargallo Moya, Antonio (1992): Los Fueros de Aragón [según el ms del Archivo Municipal de Miravete de la Sierra (Teruel)], Zaragoza, Anubar Ediciones.

Lacruz Berdejo, José Luis (1945): "Fueros de Aragón hasta 1265”, Anuario de Derecho Aragonés, II, pp. 223-363.

Lalinde Abadía, Jesús (1976): Los Fueros de Aragón, Zaragoza, Librería General.

Martínez Díez, Gonzalo (1980): "En torno a los Fueros de Aragón de las cortes de Huesca de 1247”, Anuario de Historia del Derecho Español, L, pp. 69-92.

Morales Arrizabalaga, Jesús (2007): Fueros y libertades del reino de Aragón, de su formación medieval a la crisis preconstitucional (1076-1800), Zaragoza, Rolde de Estudios Aragoneses.

Molho, Mauricio (1964): El fuero de Jaca, Zaragoza, CSIC, Escuela de Estudios Medievales, Instituto de Estudios Pirenaicos.

Pérez Martín, Antonio (1979): Fori Aragonum vom Codez von Huesca (1247) bis zur Reform Philipps II (1547), Topos Verlag, Vaduz-Liechtenstein.

Pérez Martín, Antonio (1985): "El estudio de la recepción del derecho común en España”, en Pablo Salvador Coderch y Joaquín Cerdá Ruiz-Funes (coords.), I Seminario de Historia del Derecho y derecho privado. Nuevas técnicas de investigación, Bellaterra, Universitat Autònoma de Barcelona, Servei de Publicacions, pp. 241-325.

Pérez Martín, Antonio (1999): Los Fueros de Aragón: La compilación de Huesca, Zaragoza, El justicia de Aragón.

Pérez Martín, Antonio (2010): Los Fueros de Aragón: La compilación de Huesca, Zaragoza, El justicia de Aragón.

Tilander, Gunnar (1937): Los Fueros de Aragón según el manuscrito 458 de la Biblioteca Nacional de Madrid, Lund, C. W. K. Gleerup.

Tilander, Gunnar (1956): Vidal Mayor. Traducción aragonesa de la obra In Excelsis Dei Thesauris de Vidal de Canellas, Lund, Hakan Ohlssons Boktryckeri.

Fecha de recepción: 3 de julio de 2012

Fecha de aceptación: 5 de noviembre de 2012 